\title{
Intertemporal Equilibria with Knightian Uncertainty
}

\author{
Rose-Anne Dana * Frank RIEdeL $^{\dagger}$
}

September 20, 2012

\begin{abstract}
We study a dynamic and infinite-dimensional model with incomplete multiple prior preferences. In interior efficient allocations, agents share a common risk-adjusted prior and subjective interest rate. Interior efficient allocations and equilibria coincide with those of economies with subjective expected utility and priors from the agents' multiple prior sets. A specific model with neither risk nor uncertainty at the aggregate level is considered. Risk is always fully insured. For small levels of ambiguity, there exists an equilibrium with inertia where agents also insure fully against Knightian uncertainty. When the level of ambiguity exceeds a critical threshold, full insurance no longer prevails and there exist equilibria with inertia where agents do not insure against uncertainty at all. We also show that equilibria with inertia are indeterminate.
\end{abstract}

Key words and phrases: Knightian Uncertainty, Ambiguity, Incomplete Preferences, General Equilibrium Theory, No Trade, Dynamic General Equilibrium

JEL subject classification: D51, D81, D91

* CEREMADE, UMR CNRS 7534, Université Paris IX Dauphine, Pl. de Lattre de Tassigny, 75775 Paris Cedex 16, FRANCE dana@ceremade.dauphine.fr. Rose-Anne Dana acknowledges the support of the Fondation du Risque, chaire Dauphine-ENSAE-Groupama, "Les particuliers face au risque" and of the ANR project "Risque".

${ }^{\dagger}$ Institute of Mathematical Economics, Bielefeld University and ORFE, Princeton University. We thank two referees for comments. This paper has been presented at the workshop on Economic Theory in Vigo 2009, the Bielefeld-Münster Finance Day 2010, the meeting of the "Theoretischer Ausschuss" of the Verein für Socialpolitik 2010, the European Workshop on General Equilibrium Theory in Krakow 2010, the workshop in honor of Daniel Ellsberg in Vienna 2010, and at the universities Bocconi, Cologne, Munich (LMU), and Toulouse (TSE). We thank the participants and in particular Jean-Marc Bonnisseau and Josè Faro for comments. Frank Riedel thanks University Paris IX Dauphine for hospitality during the visit in 2007 and 2009. Financial support by DFG- project Ri 1128-3-1 and International Research Training Group EBIM ("Economic Behavior and Interaction Models") is gratefully acknowledged. 


\section{Introduction}

Some contingencies have relatively stable probability distributions. Mortality rates, health care cost, and car accidents are a case in point. For others, the bankruptcy risk of an individual firm ranked "BB" to give a current example, the probabilities are unknown and rather difficult to estimate. The economic literature distinguishes between risk - where the outcomes are unknown, but the probabilities are known - and uncertainty - where even the distribution of outcomes is not known exactly. Many models of decision under uncertainty have been proposed within the past ten years, mostly with complete preferences (see Rigotti, Shannon, and Strzalecki (2008) for an overview). Models with incomplete preferences are scarcer but have raised recent interest (see, e.g., Bewley (2002), Rigotti and Shannon (2005), Nehring (2009), Faro (2010), Gilboa, Maccheroni, Marinacci, and Schmeidler (2010), Nascimento and Riella (2011), Ok, Ortoleva, and Riella (2012)).

With incomplete preferences, market clearing and utility maximization alone do not preclude situations where agents end up with a consumption bundle they cannot compare to their initial endowment. Bewley (2002) suggests to exclude such unmotivated betting by an inertia principle: agents never trade to a plan whose expected utility is not comparable to their initial endowment. The market implications of the Bewley approach in the static setting have been worked out in Rigotti and Shannon (2005) ${ }^{1}$. In this paper, we extend their analysis to a dynamic and infinite dimensional setting.

We first analyze the economy at a general equilibrium level adding new proof techniques. We then study a class of economies without aggregate uncertainty in order to highlight the differences between incomplete preferences with inertia on the one side and classic expected utility and/or pessimistic multiple prior models on the other. Equilibria with inertia are neither unique nor determinate; low ambiguity will lead to an infinite set of equilibria with inertia almost fully insured while high ambiguity yields equilibria with inertia with little or no insurance against Knightian uncertainty.

We generalize Rigotti and Shannon's result that an allocation is efficient if and only if the agents share some marginal rate of substitution. In contrast to the static setup, the construction of risk-adjusted priors is not a trivial normalization in the dynamic context. In fact, both the risk-adjustment at

\footnotetext{
${ }^{1}$ Easley and O'Hara (2010) translate the approach into a static mean-variance world, study conditions for no trade in that setting, and relate it to the recent liquidity crisis.
} 
an allocation and the discounting induced by the marginal rates of substitution have to be distinguished in a dynamic context. To determine these two effects, one has to go backwards through the "tree" by taking the available information into account. We decompose the marginal rate of substitution into a martingale and a predictable process in order to construct the riskadjusted priors and the subjective discount rates. We show that an allocation is efficient if and only if the agents agree on a risk-adjusted prior and an interest rate. The role of interest rates is pertinent to dynamic models.

We then show that an interior allocation-price pair is an equilibrium if and only if it is an equilibrium in a corresponding economy where each agent has expected utility preferences for one specific subjective belief picked from his set of priors. As a consequence, one usually has an abundance of equilibria.

Following Rigotti and Shannon, we add inertia as an equilibrium refinement. We provide a new, shorter proof for the existence of equilibria with inertia with the help of an auxiliary utility function which might be useful in other contexts as well. This auxiliary utility function belongs to the class of variational preferences (Maccheroni, Marinacci, and Rustichini (2006)). If $\mathscr{P}^{i}$ denotes an agent's set of priors, $\omega^{i}$ her endowment and $U^{i}$ the utility index, we introduce variational preferences anchored ${ }^{2}$ at $\omega^{i}$ given by

$$
\min _{P \in \mathscr{P} i} E^{P}\left(U^{i}(x)-U^{i}\left(\omega^{i}\right)\right) .
$$

It is easy to show that an equilibrium in the economy with the above variational preferences is an equilibrium with inertia in the Bewley economy. With the help of the same auxiliary utility function, one can also study when initial endowments are the unique equilibrium allocation with inertia, generalizing a result by Rigotti and Shannon (2005).

We next analyze a model without aggregate uncertainty nor risk. When agents have the same objective or subjective priors and are risk averse, they fully insure in equilibrium, and the equilibrium allocation is unique. This result carries over to uncertainty averse agents if they share a common prior, as Billot, Chateauneuf, Gilboa, and Tallon (2000) and Dana (2002) have shown. The situation is different with incomplete preferences and inertia.

In our model, the risky part of the market is fully insured in any equilibrium. Hence, we do have trade in the part of the market where agents buy insurance against risk. When the level of ambiguity is sufficiently small,

\footnotetext{
${ }^{2}$ These preferences have, independently, been axiomatized and studied by Mihm (2010); an application to development economics can be found in Bryan (2010).
} 
the full insurance equilibrium - where agents also insure the uncertain part of their endowment fully - is an equilibrium with inertia. As soon as ambiguity exceeds a critical threshold, this holds no longer true. With high ambiguity, each agent has some very optimistic prior in his belief set; under this prior, the agent prefers the uncertain part of his endowment to the full insurance allocation. The inertia constraint - which requires that the agent trades only if the new consumption plan is preferred to the endowment for all priors - then inhibits trade in uncertain assets. We identify a specific equilibrium where agents do not trade at all in the uncertain assets (they do trade away their risk, though) — we have a market "freeze" for insurance against uncertain events.

Inertia is not sufficient to yield uniqueness or even determinacy of equilibria. With low levels of ambiguity, in a neighborhood of the full insurance equilibrium, there are infinitely many equilibria with inertia where agents keep some uncertainty. With high levels of ambiguity, there is a continuum of equilibria with very limited trade for uncertain assets. Our example thus highlights that both with small and large levels of ambiguity, the refinement of inertia is not sufficient to yield determinate equilibria.

The paper is organized as follows. The next section sets up the model. Section 2 discusses the dynamic market with incomplete preferences and inertia in general. Section 3 contains our specific model with no aggregate uncertainty nor risk. Proofs for Section 2 are collected in the first of two appendices; the other one studies Mackey-continuity of variational preferences anchored at endowment.

\section{Efficiency, Equilibria, Inertia}

This section contains our analysis of general dynamic multiple prior economies with incomplete expected utility preferences. After setting up the model, we first characterize efficient allocations before moving on to equilibria with and without inertia. As we explain most of our results in words, we have put all proofs into Section A.1.

\section{$2.1 \quad$ Model}

We consider a pure exchange economy with $I$ agents who face Knightian uncertainty. Time is discrete from 0 to $T$ and there is one consumption good in 
each state of the world. Information is described by a filtration $\left(\mathscr{F}_{t}\right)_{t=0, \ldots, T}$ on a probability space $\left(\Omega, \mathscr{F}, P_{0}\right)$. Let $\mathscr{A}$ be the $\sigma$-field on $\Omega \times\{0, \ldots, T\}$ generated by all adapted processes, and let $\zeta$ be the uniform probability measure on $\{0, \ldots, T\}$. The commodity space is given by the set of essentially bounded, adapted processes $\mathscr{X}=L^{\infty}\left(\Omega \times\{0, \ldots, T\}, \mathscr{A}, P_{0} \otimes \zeta\right)$, and consumption plans are the nonnegative elements in $\mathscr{X}_{+}=L_{+}^{\infty}\left(\Omega \times\{0, \ldots, T\}, \mathscr{A}, P_{0} \otimes \zeta\right)$. Each agent comes with an endowment $\omega^{i}=\left(\omega_{t}^{i}\right)_{t=0, \ldots, T} \in \mathscr{X}_{+}$that is bounded away from $0 . \omega:=\sum_{i=1}^{I} \omega^{i}$ is aggregate endowment.

Agents have incomplete expected utility preferences that are induced by a set of priors (probability measures) $\mathscr{P}^{i}$ on $\left(\Omega, \mathscr{F}, P_{0}\right)$. Agent $i$ prefers consumption plan $c$ to consumption plan $d$, or $c \succeq^{i} d$, if and only if

$$
E^{Q} U^{i}(c) \geq E^{Q} U^{i}(d) \quad \text { for all priors } Q \in \mathscr{P}^{i},
$$

where the intertemporal preferences of agents are described by an additively separable utility function of the form

$$
U^{i}(c)=\sum_{t=0}^{T} u^{i}\left(t, c_{t}\right)
$$

for some continuous function $u^{i}:\{0, \ldots, T\} \times \mathbb{R}_{+} \rightarrow \mathbb{R}$ that is strictly increasing, strictly concave, and continuously differentiable in its second variable. The derived strict preference relation $\succ^{i}$ satisfies $c \succ^{i} d$ if and only if $c \succeq^{i} d$ and we have

$$
E^{Q} U^{i}(c)>E^{Q} U^{i}(d) \text { for some prior } Q \in \mathscr{P}^{i} .
$$

In order to have a short name, we call these preferences B-preferences (B for Bewley) and the corresponding economy a B-economy. We frequently compare this economy with incomplete preferences to some economy with heterogeneous priors $Q^{i} \in \mathscr{P}^{i}$, but complete preferences. We call that economy the $S$-economy with priors ${ }^{3} Q=\left(Q^{1}, \ldots, Q^{I}\right)$.

As the priors are defined on the probability space $\left(\Omega, \mathscr{F}, P_{0}\right)$, all priors $Q \in \mathscr{P}^{i}$ are absolutely continuous with respect to the reference probability ${ }^{4}$

\footnotetext{
${ }^{3}$ We prefer the name $S$-economy (for Savage economy) to the name risk economy that has been used elsewhere because we interpret the economy with priors $Q=\left(Q^{1}, \ldots, Q^{I}\right)$ as an economy under uncertainty where agents' preferences conform to Savage's axioms that allow to derive a unique prior $Q^{i}$. In a risk economy, the probabilities are objectively given, and all agents use the same prior.

${ }^{4}$ The assumption of absolute continuity is discussed in Epstein and Marinacci (2007).
} 
$P_{0}$. We assume throughout that they are even equivalent to $P_{0}$, and that $\mathscr{P}^{i}$ is weakly compact ${ }^{5}$ in the space $L^{1}\left(\Omega, \mathscr{F}, P_{0}\right)$ for all agents $i$.

\subsection{Efficient Allocations}

We study efficient allocations in B-economies. Let us start by fixing the concepts.

An allocation $x \in \mathscr{X}_{+}^{I}$ is a family of $I$ contingent consumption processes. The allocation $x=\left(x^{i}\right)_{i=1, \ldots, I}$ is feasible if $\sum x^{i}=\omega$. It is efficient if it is feasible and there is no other feasible allocation $y=\left(y^{i}\right)_{i=1, \ldots, I}$ such that $y^{i} \succeq^{i} x^{i}$ for all agents $i=1, \ldots, I$ and $y^{i} \succ^{i} x^{i}$ for some $i$.

In order to prepare our main results we discuss now the marginal rates of substitution. In contrast to Rigotti and Shannon's static setup, the construction of risk-adjusted priors is not a trivial normalization in the dynamic context. In fact, both the risk-adjustment at an allocation and the discounting induced by the marginal rates of substitution have to be distinguished in a dynamic context. To determine these two effects, one has to go backwards through the "tree" by taking the available information into account. We will use a Doob-type martingale decomposition to identify the risk-adjusted prior and the subjective interest rates.

Fix a prior $Q^{i} \in \mathscr{P}^{i}$ for every agent $i$. We denote by $\left(q_{t}^{i}\right)$ the density process of $Q$ with respect to $P_{0}$. We can rewrite the utility with prior $Q^{i}$ as a state-dependent expected utility function with respect to $P_{0}$ :

$$
E^{Q^{i}} U^{i}\left(x^{i}\right)=E^{Q^{i}} \sum_{t=0}^{T} u^{i}\left(t, x_{t}^{i}\right)=E^{P_{0}} \sum_{t=0}^{T} u^{i}\left(t, x_{t}^{i}\right) q_{t}^{i} .
$$

The marginal rate of substitution between date 0 and date $t$ is given by

$$
M R S_{t}^{i}\left(x^{i}, Q^{i}\right)=\frac{u_{x}^{i}\left(t, x_{t}^{i}\right) q_{t}^{i}}{u_{x}^{i}\left(0, x_{0}^{i}\right)}
$$

We denote the set of all (processes of) marginal rates of substitution at some consumption plan $x^{i}$ by

$$
\Psi^{i}\left(x^{i}\right)=\left\{M R S^{i}\left(x^{i}, Q^{i}\right) \mid Q \in \mathscr{P}^{i}\right\} .
$$

\footnotetext{
${ }^{5}$ By the Dunford-Pettis theorem, this assumption is satisfied for closed convex sets $\mathscr{P}^{i}$ if the densities in $\mathscr{D}$ are bounded by a $P_{0}$-integrable random variable. In particular, the assumption is satisfied for closed convex sets $\mathscr{P}^{i}$ whenever the state space $\Omega$ is finite.
} 
One can use the marginal rates of substitution to define a pricing probability $Q^{i}\left(x^{i}\right)$ — the so-called risk-adjusted probability — for agent $i$ and a subjective interest rate process $r_{t}^{i}$ that defines a price functional supporting efficient allocations. They are defined in such a way that we have

$$
E^{Q^{i}} \sum_{t=0}^{T} M R S_{t}^{i}\left(x^{i}, Q^{i}\right) x_{t}=E^{Q^{i}\left(x^{i}\right)} \sum_{t=0}^{T} \exp \left(-\sum_{u=1}^{t} r_{s}^{i}\right) x_{t}
$$

for all $x \in \mathscr{X}$. The next lemma shows that the last equation determines the risk-adjusted prior and the subjective interest rate in a unique way.

Lemma 2.1 Let $\left(x^{i}\right)$ be a feasible, interior allocation. Then there exist predictable subjective interest rate processes $\left(r_{t}^{i}\right)_{t=1, \ldots, T, i=1, \ldots, I}$ and strictly positive $P_{0}$-martingales $\left(M_{t}^{i}\right)$ with expectation 1 such that

$$
M R S_{t}^{i}\left(x^{i}, Q^{i}\right)=M_{t}^{i} \exp \left(-\sum_{s=1}^{t} r_{s}^{i}\right)
$$

$r^{i}$ and $M^{i}$ are uniquely determined.

Note that the subjective interest rates $r^{i}$ and martingales $M^{i}$ depend both on $Q^{i}$ and $x^{i}$, so we write $r^{i}\left(Q^{i}, x^{i}\right)$ or $M^{i}\left(Q^{i}, x^{i}\right)$ to emphasize this dependence when necessary. The martingales $M^{i}$ identified in the previous lemma define a probability measure $Q^{i}\left(x^{i}\right)$ that is equivalent to $P_{0}$ and satisfies (2). These probabilities are the suitable generalization of Rigotti and Shannon's risk-adjusted priors; we denote agent $i$ 's set of risk-adjusted priors at consumption $x^{i}$ by $\Pi^{i}\left(x^{i}\right)$.

We have now collected the relevant tools to state our main theorem on interior efficient allocations. At an efficient allocation, agents share a riskadjusted prior. In contrast to the static framework of Rigotti and Shannon (2005), this condition is only necessary, but not sufficient for efficiency. Only if the agents also agree on the interest rate used to discount future consumption, the allocation is efficient. We also show that efficiency with incomplete preferences is equivalent to having efficiency in some S-economy for some choice of priors $Q^{i} \in \mathscr{P}^{i}$.

Theorem 2.2 The following assertions are equivalent for an interior allocation $x$ : 
1. $x$ is efficient,

2. the agents' sets of marginal rates of substitution intersect,

$$
\bigcap_{i=1}^{I} \Psi^{i}\left(x^{i}\right) \neq \emptyset
$$

3. the agents share a risk-adjusted prior

$$
\bigcap_{i=1}^{I} \Pi^{i}\left(x^{i}\right) \neq \emptyset
$$

and for a common risk-adjusted prior $Q \in \bigcap_{i=1}^{I} \Pi^{i}\left(x^{i}\right)$ all subjective interest rates are equal, i.e.

$$
r^{i}\left(Q, x^{i}\right)_{t}=r^{j}\left(Q, x^{j}\right)_{t}
$$

for all $i, j=1, \ldots, I$ and $t=1, \ldots, T$,

4. for some selection of priors $Q^{i} \in \mathscr{P}^{i}, i=1, \ldots, I, x$ is efficient in the $S-$ economy with priors $Q=\left(Q^{1}, \ldots, Q^{I}\right)$.

\subsection{Equilibria with and without Inertia}

We study now equilibrium allocations and prices for B-economies. There are usually infinitely many equilibria as any S-economy equilibrium leads to an equilibrium in the B-economy. We then adapt Rigotti and Shannon's version of Bewley's inertia criterion as a refinement.

A price for our economy is given by an adapted, integrable, positive process $\left(p_{t}\right) \in L^{1}\left(\Omega \times\{0, \ldots, T\}, \mathscr{A}, P_{0} \otimes \zeta\right)$. Let $\mathbb{P}$ be the set of price processes. We denote by

$$
p . x:=E^{P_{0}} \sum_{t=0}^{T} p_{t} x_{t}
$$

the linear functional associated with the price $\left(p_{t}\right)$.

A feasible allocation $x^{*} \in \mathscr{X}_{+}^{I}$ and a price $p^{*} \in \mathbb{P}$ form an equilibrium if, for every $i, p^{*} \cdot x^{* i}=p^{*} \cdot \omega^{i}$ and if $x^{i} \succ x^{* i}$ implies $p^{*} . x^{i}>p^{*} . \omega^{i}$ (there is no budget feasible consumption plan that strictly dominates $x^{* i}$ ). A feasible allocation 
$x^{*} \in \mathscr{X}_{+}^{I}$ and a price $p^{*} \in \mathbb{P}$ is a equilibrium with transfer payments if $x^{i} \succ x^{* i}$ implies $p^{*} \cdot x^{i}>p^{*} \cdot x^{* i}$ for all $i$.

Before we start our analysis, let us remark that the first welfare theorem trivially holds true for a B-economy ${ }^{6}$. A weak ${ }^{7}$ form of the second welfare theorem follows from the next proposition. It also proves existence of equilibria without needing any abstract general existence theorem.

Proposition 2.3 1. Let $\left(x^{*}, p^{*}\right)$ be an equilibrium for an S-economy with priors $Q^{i} \in \mathscr{P}^{i}, i=1, \ldots, I$. Then $\left(x^{*}, p^{*}\right)$ is an equilibrium in the B-economy. Similarly, any equilibrium with transfer payments for an S-economy with priors $Q^{i} \in \mathscr{P}^{i}, i=1, \ldots, I$ is an equilibrium with transfer payments in the B-economy.

2. Any interior efficient allocation $\left(x^{*}\right)$ is the allocation of a equilibrium with transfer payments.

3. Any interior equilibrium $\left(x^{*}, p^{*}\right)$ in the B-economy is an interior equilibrium for an $S$-economy with priors $Q^{i} \in \mathscr{P}^{i}, i=1, \ldots, I$.

Let us apply the above result to economies without aggregate uncertainty (where the aggregate endowment is a deterministic process). It is well known that in S-economies with homogenous priors, agents fully insure in equilibrium. Combined with the previous proposition, we obtain an existence result for full insurance equilibria in B-economies. In our dynamic setting, a full insurance allocation $x \in \mathscr{X}_{+}^{I}$ is a family of $I$ deterministic consumption processes (that may depend on time, but not on states of the world).

Corollary 2.4 Assume that there exists no aggregate uncertainty and that agents share at least one prior,

$$
\bigcap_{i=1}^{I} \mathscr{P}^{i} \neq \emptyset .
$$

Then there exists an equilibrium allocation with full insurance.

\footnotetext{
${ }^{6}$ Indeed let $\left(x^{*}, p^{*}\right)$ be an equilibrium. If $x^{*}$ is not efficient, then from Lemma A.2, there exist $y$ feasible such that $y^{i} \succ^{i} x^{* i}$ for all $i$. But then we must have $p^{*} \cdot y^{i}>p^{*} \cdot \omega^{i}$ for all $i$, contradicting Walras law.

${ }^{7}$ As it requires interior allocations.
} 
It also follows from Proposition 2.3 that the set of equilibria is monotone with respect to the set of priors: the larger the set of priors, the larger is the set of equilibria.

Proposition 2.3 also shows that one usually has a plethora of B-equilibria as every equilibrium of some S-economy is an equilibrium. It is plausible that this leads typically to a continuum of equilibria when the sets of priors are not singletons. Therefore, indeterminacy of equilibrium allocations and prices is the rule, not the exception for B-economies ${ }^{8}$.

On the other hand, many of these equilibria lead to consumption plans that the agents cannot compare with their endowment because they have incomplete preferences. In this case, one might well ask why these agents should decide to take these plans in the first place. Following in spirit Rigotti and Shannon ${ }^{9}$, we impose the additional condition of inertia. An equilibrium $\left(x^{*}, p^{*}\right)$ satisfies the inertia condition if for all agents $i$ with $x^{* i} \neq \omega^{i}$, we have $x^{* i} \succ^{i} \omega^{i}$.

Given the additional constraint, it is no longer obvious that equilibria satisfying this refinement exist. Rigotti and Shannon provide a sophisticated proof based on Gale and Mas-Colell's (1975) fixed point theorem to establish existence. We provide here a simple and shorter proof for existence of equilibria with inertia based on an auxiliary economy with complete static variational preferences as axiomatized in Maccheroni, Marinacci, and Rustichini (2006). We construct uncertainty-averse preferences such that any equilibrium of the auxiliary economy with those preferences is an equilibrium with inertia.

Definition 2.5 We call a utility function of the form

$$
V^{i}(x)=\min _{Q \in \mathscr{P} i} E^{Q}\left(\left(U^{i}(x)-U^{i}\left(\omega^{i}\right)\right)\right)
$$

variational utility anchored at $\omega^{i}$.

An agent of the above type compares the expected gain from moving away from her endowment $\omega^{i}$ to the new consumption plan $x^{i}$. Variational utility

\footnotetext{
${ }^{8}$ A detailed analysis of indeterminacy in the static setting is in Rigotti and Shannon (2005).

${ }^{9}$ Our definition of inertia is slightly different from Rigotti and Shannon's because we require strict preference of the new consumption bundle over the status quo whereas Rigotti and Shannon use only weak preference (see p. 210 in their paper).
} 
functions anchored at $\omega^{i}$ belong to the class of variational preferences that generalize the Gilboa-Schmeidler preferences; they have been axiomatized ${ }^{10}$ in Maccheroni, Marinacci, and Rustichini (2006).

In our case, these (complete) preferences are useful because equilibria in economies with such agents are also equilibria with inertia in our B-economy.

Theorem 2.6 Any equilibrium of an economy with variational utilities anchored at endowments $V^{i}(x)=\min _{Q \in \mathscr{P}^{i}} E^{Q}\left(\left(U^{i}(x)-U^{i}\left(\omega^{i}\right)\right)\right)$ is an equilibrium with inertia. In particular, equilibria with inertia exist.

Remark 2.7 In the previous theorem, the specific form of the auxiliary utility function is not important. We could as well have used

$$
\tilde{V}^{i}(x)=\min _{Q \in \mathscr{P}^{i}} \frac{E^{Q} U^{i}(x)}{E^{Q} U^{i}\left(\omega^{i}\right)},
$$

for all agents, e.g., or even $V^{i}$ for some agents, and $\tilde{V}^{i}$ for others, leading to other equilibria with inertia, in general. From the economic point of view, the crucial point is to have a dependence on endowment. The important point on the technical side is Mackey-continuity of the employed utility functional.

Inertia is a very strict requirement, and can lead to market breakdown in the sense that the initial endowment is the unique equilibrium allocation. Our above argument leads easily to a characterization of such no trade situations.

Corollary $2.8\left(\omega^{i}\right)$ is the unique equilibrium allocation with inertia if and only if $\left(\omega^{i}\right)$ is a no trade equilibrium allocation in the economy with complete preferences given by variational utilities anchored at endowments as in Equation (3).

\section{A Model without Aggregate Uncertainty Nor Risk}

Several authors have already pointed out, through no-trade results, that ambiguity modeled with Bewley preferences could explain the breakdown of

\footnotetext{
${ }^{10}$ In order to get the variational representation for our anchored utilities, one has to introduce the cost or penalty function on the set $\Delta$ of all probability measures. One has to set $c(P)=\infty$ for $P \notin \mathscr{P}^{i}$ and $c(P)=-E^{P} U^{i}\left(\omega^{i}\right)$ for $P \in \mathscr{P}^{i}$.
} 
markets. Moreover Rigotti and Shannon have proved that if no trade is an equilibrium, then it is the unique equilibrium with inertia. This raises the following natural questions. How robust are these properties? Suppose that there is risk and uncertainty. Is it true that for ambiguity large enough, there will be a breakdown of the uncertainty market? Can we provide conditions for equilibrium with inertia to be unique?

We address these questions in an economy without aggregate uncertainty whose individual endowments are subject to both a risky and an uncertain source.

As is well known, with expected utility and homogenous priors, all risk and uncertainty is fully insured. The equilibrium is also unique in that case. The same holds true for pessimistic multiple prior preferences if agents share at least one prior (Billot, Chateauneuf, Gilboa, and Tallon (2000), although prices need not be determinate).

In the B-economy,

- risk is completely insured, and thus there is always trade,

- if ambiguity is small, full insurance is a robust equilibrium: for any $\varepsilon>0$, there are infinitely many equilibria with inertia with agents'consumptions bounded by $\varepsilon$,

- when ambiguity exceeds a certain critical level, the full insurance allocation (where also the uncertain part of the endowment is fully insured) is not an equilibrium with inertia,

- in this case, there is an equilibrium with inertia where subjective uncertainty is not traded at all and there are infinitely many equilibria with inertia with very little trading of uncertainty around this equilibrium,

- finally, although inertia is a strong equilibrium refinement, we nevertheless have indeterminacy of equilibria with inertia ${ }^{11}$.

We now introduce the formal model. The basic building block is a probability space $\left(\Omega, \mathscr{F}, P_{0}\right)$. Whenever we write the expectation operator $E$ below, we mean the expectation under our reference measure $P_{0}$.

\footnotetext{
${ }^{11}$ Bewley (2002) sketches the lack of insurance incentives and the possibility of no trade equilibria in Section 6 of his paper. No trade equilibria are also discussed in Rigotti and Shannon (2005). Here, we do always have trade (in the risk market), and a market freeze in the uncertain part of the market.
} 
On $\left(\Omega, \mathscr{F}, P_{0}\right)$, there are two independent random walks

$$
R_{t}=\sum_{s=1}^{t} \rho_{s} \quad \text { and } \quad U_{t}=\sum_{s=1}^{t} \nu_{s}
$$

for $t \geq 1$ and $R_{0}=U_{0}=0$. Under our reference measure $P_{0}$, the $\left(\rho_{t}\right)$ and $\left(\nu_{t}\right)$ are independent and identically distributed with common distribution $F=N(0,1)$, the standard normal distribution.

The information filtration is the natural filtration generated by the two processes $R$ and $U$, i.e.

$$
\mathscr{F}_{t}=\sigma\left(R_{1}, \ldots, R_{t}, U_{1}, \ldots, U_{t}\right)
$$

We also introduce the information generated by $U$ alone,

$$
\mathscr{F}_{t}^{U}=\sigma\left(U_{1}, \ldots, U_{t}\right)
$$

Let us assume that there are two agents who use the same class of priors $\mathscr{P}$. The priors $Q \in \mathscr{P}$ are such that all agents agree that the $\left(\rho_{t}\right)$ are standard normal and independent of $U$.

In contrast, agents are uncertain about the distribution of the $\nu_{t}$ that generate the random walk $U$. For the sources of uncertainty $\nu_{t}$, we model the idea that they come from identical experiments, but we use multiple priors. One way to do this in such a way that we can later compare the results with ambiguity-averse decision makers is to use the following model of "independent experiments with identical ambiguity" 12 .

The family of priors is defined by their density processes with respect to $P_{0}$; they are of the form

$$
q_{t}=\exp \left(\sum_{s=1}^{t}\left(\alpha_{s} \nu_{s}-\frac{1}{2} \alpha_{s}^{2}\right)\right)
$$

\footnotetext{
${ }^{12}$ This model is discussed at length in Epstein and Schneider (2003) where it is called "independent and indistinguishably distributed". We use the name coined by Riedel (2009). For Gilboa-Schmeidler preferences, time-consistency is an issue. Indeed, not every convex set of priors leads to time-consistent preferences. Epstein and Schneider have identified the property of rectangularity, or as it has been called elsewhere, stability under pasting, as necessary and sufficient for time-consistency. Our set of priors is rectangular.
} 
for some $\mathscr{F}^{U}$-predictable process $\left(\alpha_{t}\right)$ with values in the interval $[-\kappa, \kappa]$ for some $\kappa>0^{13}$. Note that $\alpha_{t}$ depends only on the values of $U_{1}, \ldots, U_{t-1}$ as it is $\mathscr{F}^{U}$-predictable. Note also that $\left(\rho_{t}\right)$ are standard normal under all priors with such density processes (as the density depends only on $U$ ) and independent of $U$. It is also worthwhile to remark that the set of densities that we use here is not convex ${ }^{14}$.

We assume that the two agents have period utilities

$$
u^{i}(t, c):=\exp (-\rho t) v^{i}(c):=-\exp (-\rho t-c)
$$

for some subjective discount $\operatorname{rate}^{15} \rho$. Agents thus have constant absolute risk aversion 1.

Let endowments be

$$
\omega_{t}^{1}=R_{t}+U_{t} \quad \text { and } \quad \omega_{t}^{2}=-\omega_{t}^{1} .
$$

Aggregate endowment is thus zero, or one can say we look at net trades. the the arguments go through with a deterministic aggregate endowment $K_{t} 0$ in period $t$; as we are in a framework with normal distributions and constant absolute risk aversion, the constant $K_{t}$ cancels in the calculations ${ }^{16}$.

Initially, both agents are affected by risk and uncertainty. As there is no aggregate risk (nor uncertainty), one might expect rational agents to insure perfectly in equilibrium. This is indeed the case for Gilboa-Schmeidler preferences; we recall here the result of Billot, Chateauneuf, Gilboa, and Tallon (2000) and Dana (2002) that translates easily to the dynamic setting.

\footnotetext{
${ }^{13}$ Readers familiar with the continuous-time literature recognize here the usual Girsanov transform for a change of measure; our model is thus a discrete-time counterpart of $\kappa^{-}$ ambiguity as in Chen and Epstein (2002), except that we have both risk and uncertainty, and the risky part does not appear in the density (because its distribution has to remain the same for all priors)

${ }^{14}$ In order to fully mimic the results of the preceding section, one would thus have to pass to the closed convex hull of this set of priors. Note that, by linearity of the expectation operator, the closed convex hull induces the same preference relation as the original class. We do not develop this further here because it is not the main point of this section.

${ }^{15}$ The case of different discount rates leads to the same results. Here, a (deterministic) trade pattern appears to the different degrees of time preferences. As we are interested in the insurance aspect, we take homogenous discount rates.

${ }^{16} \mathrm{We}$ allow here for negative consumption and take the commodity space to be the space of all square-integrable adapted processes. Even though this does not fit into our general framework of the previous subsection, the basic results carry over. We give details below when needed.
} 
Proposition 3.1 In every equilibrium of the Gilboa-Schmeidler economy where preferences are given by

$$
\min _{Q \in \mathscr{P}} E^{Q} \sum_{t=0}^{T} v^{i}\left(t, c_{t}^{i}\right),
$$

there is full insurance.

Let us now come to the B-economy. Our first point is that equilibria are independent of $R$. To this end, we show that any allocation which contains $R$ in a nontrivial way is strictly dominated by its conditional expectation with respect to the $\sigma$-field generated by $\left(U_{1}, \ldots, U_{t}\right)$.

Theorem 3.2 In every equilibrium $(x, p)$ of the B-economy, risk is fully insured in the sense that the equilibrium allocation $x$ depends only on $U$ not on $R$.

Proof: The fact that risk is completely insured is due to the fact that agents share a common prior for the distribution of the risky random walk $R$. Whenever there is a feasible allocation $x=\left(x^{1}, x^{2}\right)$ that contains $R$ in a nontrivial way, agents can pass to the corresponding (conditional) expectation $y_{t}^{1}=E\left[x_{t}^{1} \mid U_{1}, \ldots, U_{t}\right], y_{t}^{2}=E\left[x_{t}^{2} \mid U_{1}, \ldots, U_{t}\right]$. Note that we do not use different priors here because the distribution of $R$ is the same under every prior. As there is no aggregate uncertainty, the allocation $y=\left(y^{1}, y^{2}\right)$ is still feasible, and by risk aversion, both agents are better off.

We next describe a subset of the set of all equilibria, namely those which are obtained as equilibria of Savage economies with heterogeneous priors picked in agents' sets of priors. There are even more equilibria than the ones constructed here because one can also form convex combinations of our priors. However the subset we describe is large enough for the indeterminacy argument we shall develop.

Proposition 3.3 Let $\left(\alpha_{s}\right),\left(\beta_{s}\right)$ be two $\mathscr{F}^{U}$-predictable processes with values in $[-\kappa, \kappa]$. The allocations $(c,-c)$ with

$$
c_{t}-c_{0}=\frac{1}{2}\left(\sum_{s=1}^{t}\left(\alpha_{s}-\beta_{s}\right) \nu_{s}+\frac{\beta_{s}^{2}-\alpha_{s}^{2}}{2}\right)
$$


and the price

$$
p_{t}=\exp \left(-\rho t+\frac{1}{2}\left(\sum_{s=1}^{t}\left(\alpha_{s}+\beta_{s}\right) \nu_{s}+\frac{\beta_{s}^{2}+\alpha_{s}^{2}}{2}\right)\right) .
$$

form an equilibrium for a properly chosen $c_{0}$.

In particular for $|\alpha| \leq \kappa$, the allocation $\left(\alpha U_{t},-\alpha U_{t}\right)$ with the deterministic price $p_{t}=\exp \left(-\left(\rho+1 / 2 \alpha^{2}\right) t\right)$ is an equilibrium.

Proof: Let $\left(q_{t}^{1}\right),\left(q_{t}^{2}\right)$ be two priors' density processes for the two agents defined respectively by $\left(\alpha_{t}\right),\left(\beta_{t}\right)$. Let $\left(c_{t}\right)$ be a consumption process for agent 1 . If the marginal rates of substitution between time 0 and time $t$ of the agents are equal,

$$
\exp \left(-\rho t-\left(c_{t}-c_{0}\right)\right) q_{t}^{1}=\exp \left(-\rho t+\left(c_{t}-c_{0}\right)\right) q_{t}^{2}
$$

then the allocation $(c,-c)$ is efficient. Equivalently $c_{t}-c_{0}=\frac{1}{2} \log \frac{q_{t}^{1}}{q_{t}^{2}}$ and we obtain (5). The common marginal rate of substitution being a supporting price, we have $p_{t}=\exp \left(-\rho t-\left(c_{t}-c_{0}\right)\right) q_{t}^{1}=\exp (-\rho t) \sqrt{q_{t}^{1} q_{t}^{2}}$ and therefore we obtain (6). From the equilibrium budget constraint, we must finally have

$$
\sum_{s=0}^{T} E\left[p_{t}\left(U_{t}+R_{t}-c_{0}-\frac{1}{2} \log \frac{q_{t}^{1}}{q_{t}^{2}}\right)\right]=0 .
$$

(7) determines $c_{0}$. The corresponding equilibrium consumption process is obtained from (5).

When $\alpha_{s}=-\beta_{s}=\alpha$, (5) yields $c_{t}-c_{0}=\alpha U_{t}$. The corresponding price process $p_{t}=\exp \left(-\left(\rho+1 / 2 \alpha^{2}\right) t\right)$ is a deterministic process. As $E\left(R_{t}\right)=$ $E\left(U_{t}\right)=0$ and $E \log \frac{q_{t}^{1}}{q_{t}^{2}}=E\left(2 \alpha U_{t}\right)=0$, we obtain $c_{0}=0$ from $(7)$.

Remark 3.4 (i) The above proposition highlights an interesting indeterminacy of the interest rate in equilibrium. The supporting price process for the above equilibria implies an interest of $\rho+\frac{\alpha^{2}}{2}$ for $\alpha \in[-\kappa, \kappa]$. The interest rate is thus quite indeterminate, with interesting implications for the term structure. We will see below that the indeterminacy of the interest rate, while reduced by the inertia condition, persists.

(ii) Clearly the equilibria constructed above are independent of $R$. 
It follows from Proposition 3.3 that there is a plethora of equilibrium allocations in $\mathrm{B}$-economies. We shall now on focus on two specific equilibria, the full insurance allocation $x^{1}=x^{2}=0$ (choose $\alpha=\beta=0$ e.g.) and the equilibrium with no insurance of uncertainty $(\alpha=\beta=1)$.

Let us first prove a useful lemma.

Lemma 3.5 Let $Q$ be an agent's prior associated to the process $\left(\alpha_{s}\right)$. Then

$$
\exp \left(\left(\frac{\gamma^{2}}{2}+\kappa|\gamma|\right) t\right) \geq E^{Q} \exp \left(-\gamma U_{t}\right) \geq \exp \left(\left(\frac{\gamma^{2}}{2}-\kappa|\gamma|\right) t\right)
$$

We are now in the position to study the inertia property for our two focal equilibria. We start with the full insurance allocation.

Theorem 3.6 For sufficiently small levels of ambiguity, namely $\kappa \leq 1$, the full insurance allocation $x^{1}=x^{2}=0$ is an equilibrium allocation with inertia. If ambiguity is sufficiently large, $\kappa>1$, the full insurance allocation $x^{1}=$ $x^{2}=0$ is not an equilibrium allocation with inertia.

Proof: From Proposition 3.3, the full insurance allocation $x^{1}=x^{2}=0$ is an equilibrium of the $\mathrm{B}$-economy. Let us first prove that the inertia condition is verified if $\kappa<1$. Let us show that for every $Q \in \mathscr{P}$ and $t=1, \ldots, T$

$$
-1>-E^{Q}\left(\exp \left(-R_{t}\right)\right) E^{Q}\left(\exp \left(-U_{t}\right)\right)=-\exp \left(\frac{t}{2}\right) E^{Q}\left(\exp -U_{t}\right)
$$

Indeed from (8),

$$
E^{Q}\left(\exp \left(-U_{t}\right)\right) \geq \exp \left(\left(\frac{1}{2}-\kappa\right) t\right)>\exp \left(-\frac{t}{2}\right)
$$

If $\kappa=1$, from $(8), E^{Q}\left(\exp \left(-U_{t}\right)\right) \geq \exp \left(-\frac{t}{2}\right)$ for every $Q \in \mathscr{P}$ and $t=$ $1, \ldots, T$, hence

$$
-1 \geq-E^{Q}\left(\exp \left(-R_{t}\right)\right) E^{Q}\left(\exp \left(-U_{t}\right)\right) .
$$

The strict inequality holds true under $P_{0}$ since $R_{t}$ and $U_{t}$ and normally distributed with mean 0 and the utility is strictly concave. Hence for $\kappa=1$, the full insurance allocation is an equilibrium allocation with inertia. 
Finally if $\kappa>1$, we verify that the inertia condition is not satisfied. Consider the prior with density

$$
q_{t}=\exp \left(\kappa U_{t}-\frac{\kappa^{2}}{2} t\right) .
$$

The period utility at date $t \geq 1$ of endowment under this prior for agent 1 is then

$$
\begin{aligned}
& -E \exp \left(-R_{t}-U_{t}+\kappa U_{t}-\frac{\kappa^{2}}{2} t\right) \\
& =-\exp -\left(\frac{\kappa^{2} t}{2}\right) E \exp \left(-R_{t}\right) E \exp \left((\kappa-1) U_{t}\right) \\
& \left.=-\exp \left(\frac{1}{2} t+\frac{1}{2}(\kappa-1)^{2} t-\frac{\kappa^{2} t}{2}\right)\right)=-\exp ((1-\kappa) t)>-1 .
\end{aligned}
$$

Hence, agent 1's inertia condition is not satisfied.

With large ambiguity, agents have a very optimistic prior for the uncertain part $U$ in their belief set. Under this prior, the average value of $U_{t}$ is so high that they would like to keep it. This offsets their sufferings from keeping the risky part $R_{t}$.

Theorem 3.6 raises a number of questions: what kind of equilibria with inertia do we get if ambiguity is large? Is the inertia refinement strong enough to yield determinacy (or uniqueness) of an equilibrium? To answer the first question, we point out a particular equilibrium with inertia that we consider to be natural: the market for risk works perfectly in the sense that agents are fully insured against risk, and there is a freeze on the market for uncertainty.

Theorem 3.7 If ambiguity is sufficiently large, $\kappa \geq 1$, the B-economy has an equilibrium with inertia $(x, p)$ with allocation

$$
x_{t}^{1}=U_{t}=-x_{t}^{2}
$$

and equilibrium price

$$
p_{t}=\exp (-(\rho+1 / 2) t)
$$

ProOF: As $\kappa \geq 1$, from Proposition 3.3, $(x, p)$ is an equilibrium. We now show that the inertia condition is satisfied. For every prior $Q \in \mathscr{P}$ we have by the usual risk aversion (concavity) argument that $E^{Q} v^{i}\left(U_{t}+R_{t}\right)=$ 
$-E^{Q}\left[\exp \left(-U_{t}\right) E^{Q}\left[\exp \left(-R_{t}\right)\right]\right]<-E^{Q}\left[\exp \left(-U_{t}\right)\right]$ because $R_{t}$ is independent of $U$ under all priors and normally distributed with mean 0 under all priors $Q \in \mathscr{P}$.

We conclude that the market for insurance of uncertainty can break down if agent use the inertia criterion in combination with incomplete expected utility preferences. This may not seem a new result since several authors have pointed out this phenomenon and discussed the possibility of no-trade for Bewley preferences. Note, however, that we do not have a no trade situation here. Agents do trade the risky parts of their endowments.

As a last point in our paper, we now demonstrate that inertia is not sufficient to determine locally unique equilibria and that the two equilibria that we focused on are robust: for $0<\kappa<1$, in any neighborhood of full insurance there are infinitely many equilibria where agents are almost fully insured, and for $\kappa \geq 1$, in any neighborhood of the no insurance of uncertainty equilibrium, there are infinitely many equilibria where there is a small trade of uncertainty.

Theorem 3.8 The equilibria with inertia are indeterminate.

For $0<\kappa<1$, there exists a continuum of equilibria with inertia with allocation $\left(c^{1},-c^{1}\right)$ and $c^{1}=\epsilon U$ for sufficiently small $\epsilon \neq 0$.

For $\kappa \geq 1$, there exists a continuum of equilibria with inertia with allocation $\left(c^{1},-c^{1}\right)$ and $c^{1}=(1-\epsilon) U$ for sufficiently small $\epsilon \neq 0$.

Proof: From Proposition 3.3, for $|\alpha| \leq \kappa$, the allocation $\left(\alpha U_{t},-\alpha U_{t}\right)$ with the deterministic price $p_{t}=\exp \left(-\left(\rho+1 / 2 \alpha^{2}\right) t\right)$ is an equilibrium. It remains to verify the inertia conditions. To this end, let us show that

$$
E^{Q} \exp \left(-U_{t}\right) \exp \left(\frac{t}{2}\right) \geq E^{Q} \exp \left(-\alpha U_{t}\right)
$$

for all $Q \in \mathscr{P}$ and $t \geq 1$. From Lemma 3.5, $E^{Q} \exp \left(-U_{t}\right) \geq \exp ((1 / 2-\kappa) t)$ and $E^{Q} \exp \left(-\alpha U_{t}\right) \leq \exp \left(1 / 2 \alpha^{2}+\kappa|\alpha|\right)$. Hence (10) is verified if

$$
1-\kappa \geq 1 / 2 \alpha^{2}+\kappa|\alpha| .
$$

For any given $\kappa<1,(11)$ is verified by $\alpha=0$ with a strict inequality, hence by continuity of the map $\alpha \rightarrow 1 / 2 \alpha^{2}+\kappa|\alpha|$ it is verified in a neighborhood of $\alpha=0$. The inertia condition for agent 2 is proved in a similar way. 
Now let us look at the case $\kappa \geq 1$. We claim that there exists an $\epsilon>0$ such that for all $\gamma \in(1-\epsilon, 1+\epsilon)$ the inertia condition is satisfied for both agents.

Pick any prior $Q \in \mathscr{P}$ and denote the density with respect to $P_{0}$ by $q$. By Lemma A.9, there exists $M>0$ sich that $E q_{t}^{2} \leq M$ for all $t=1, \ldots, T$. With the help of Hölder's inequality, we get

$$
\begin{aligned}
\left|E^{Q}\left(\exp \left(-\gamma U_{t}\right)-\exp \left(-U_{t}\right)\right)\right| & =\left|E q_{t}\left(\exp \left(-\gamma U_{t}\right)-\exp \left(-U_{t}\right)\right)\right| \\
\text { Hölder } & \leq\left(E q_{t}^{2}\right)^{1 / 2}\left(E\left(\exp \left(-\gamma U_{t}\right)-\exp \left(-U_{t}\right)\right)^{2}\right)^{1 / 2} \\
& \leq \sqrt{M}\left(E\left(\exp \left(-\gamma U_{t}\right)-\exp \left(-U_{t}\right)\right)^{2}\right)^{1 / 2}
\end{aligned}
$$

As $U$ is normally distributed under the reference measure $P_{0}$, the last expression can be computed explicitly. We have

$$
\begin{aligned}
& E\left(\exp \left(-\gamma U_{t}\right)-\exp \left(-U_{t}\right)\right)^{2} \\
& \quad=E\left(\exp \left(-2 \gamma U_{t}\right)-2 \exp \left(-(1+\gamma) U_{t}\right)+\exp \left(-2 U_{t}\right)\right) \\
& \quad=\exp \left(2 \gamma^{2} t\right)-2 \exp \left((1+\gamma)^{2} / 2 t\right)+\exp (2 t)=: f(\gamma)^{2}
\end{aligned}
$$

Note that $f$ is continuous in $\gamma$ with $f(1)=0$.

Let us finally look at the ratio $E^{Q} \exp \left(-\gamma U_{t}\right) / E^{Q} \exp \left(-U_{t}\right)$. With the help of the above estimate, we have

$$
\begin{aligned}
E^{Q} \exp \left(-\gamma U_{t}\right) / E^{Q} \exp \left(-U_{t}\right) & \leq \frac{E^{Q} \exp \left(-U_{t}\right)+\sqrt{M} f(\gamma)}{E^{Q} \exp \left(-U_{t}\right)} \\
& =1+\frac{\sqrt{M} f(\gamma)}{E^{Q} \exp \left(-U_{t}\right)}
\end{aligned}
$$

$$
\text { Lemma } 3.5 \leq 1+\sqrt{M} f(\gamma) \exp ((\kappa-1 / 2) t)
$$

For $\gamma$ sufficiently close to 1 , the last expression is strictly smaller than $\exp (t / 2)$. The inertia inequality follows, and we are done. The proof for agent 2 runs along the same lines.

\section{Conclusion}

This paper extends the analysis of economies with incomplete multiple prior preferences to dynamic and infinite-dimensional settings. For every selection 
of priors, the corresponding equilibria of the Savage economy are equilibria of our economy. The converse holds also true if the set of priors is convex and (weakly) compact. Agents thus share a common marginal rate of substitution, as in Rigotti and Shannon (2005). In economic terms, this implies more than merely sharing a risk-adjusted prior as in the static case; dynamic efficiency implies that the agents share also a common discount rate. We have a new proof of existence of equilibria with inertia that relies on an interesting tool that we call variational preferences anchored at endowment. These preferences have, independently, been axiomatized and studied by Mihm (2010).

In a specific model with normally distributed returns and constant absolute risk aversion, we obtain more results. In that economy, which contains risk and uncertainty on the individual level, but neither risk nor uncertainty at the aggregate, agents always insure against risk. For small levels of ambiguity, full insurance is an equilibrium with inertia while it is not if ambiguity crosses a critical threshold. Instead, there appears an equilibrium with inertia where trade breaks down on the uncertainty part of the market.

Although inertia is a strong equilibrium refinement, we nevertheless have indeterminacy of equilibria with inertia. This shows that Bewley's original idea is not sufficient to yield locally unique equilibria in general.

\section{A Appendix}

\section{A.1 Proofs for Section 2}

This section contains additional details and the proofs for Section 2 .

It is often convenient to work with the weak notion of efficiency. An allocation $x=\left(x^{i}\right)_{i=1, \ldots, I}$ is weakly efficient if it is feasible and there is no other feasible allocation $y=\left(y^{i}\right)_{i=1, \ldots, I}$ such that $y^{i} \succ^{i} x^{i}$ for all agents $i=1, \ldots, I$. The following technical result will turn out to be useful. Our concept of strict preference $y \succ^{i} x$ allows for the possibility that the expected utility of $y$ is the same than that of $x$ under some priors. With our assumptions on utility functions, it is enough to check for strict inequalities here.

Lemma A.1 An allocation $x=\left(x^{i}\right)_{i=1, \ldots, I}$ is weakly efficient if it is feasible and there is no other feasible allocation $y=\left(y^{i}\right)_{i=1, \ldots, I}$ such that $E^{Q} U^{i}\left(y^{i}\right)>$ $E^{Q} U^{i}\left(x^{i}\right)$ for all priors $Q \in \mathscr{P}^{i}$ for all agents $i=1, \ldots, I$. 
Proof: Clearly if $x$ is weakly efficient, there is no other feasible allocation $y=\left(y^{i}\right)_{i=1, \ldots, I}$ such that $E^{Q} U^{i}\left(y^{i}\right)>E^{Q} U^{i}\left(x^{i}\right)$ for all priors $Q \in \mathscr{P}^{i}$ for all agents $i=1, \ldots, I$. Conversely, assume that there is no other feasible allocation $y=\left(y^{i}\right)_{i=1, \ldots, I}$ such that $E^{Q} U^{i}\left(y^{i}\right)>E^{Q} U^{i}\left(x^{i}\right)$ for all priors $Q \in$ $\mathscr{P}^{i}$ for all agents $i=1, \ldots, I$ and there exists another feasible allocation $y=$ $\left(y^{i}\right)_{i=1, \ldots, I}$ such that $y^{i} \succ x^{i}$ for all agents $i=1, \ldots, I$. Since $y^{i} \neq x^{i}$ and $U^{i}$ is strictly concave and priors are equivalent to $P_{0}, E^{Q} U^{i}\left(\frac{y^{i}+x^{i}}{2}\right)>E^{Q} U^{i}\left(x^{i}\right)$ for all priors $Q \in \mathscr{P}^{i}$ and agents $i=1, \ldots, I$. As the allocation $\frac{x+y}{2}$ is feasible, we obtain a contradiction to the weak efficiency of $x$.

With a compact set of priors and strictly concave Bernoulli utility functions $U^{i}$, interior weakly efficient allocations are also efficient. We recall that in our context, an allocation is interior if it is uniformly bounded away from zero. The argument follows the usual lines, with some technical twinkles as we are in an infinite-dimensional context.

Lemma A.2 Efficient allocations are weakly efficient. Interior, weakly efficient allocations are efficient.

Proof: Clearly if $x$ is efficient, it is weakly efficient. Conversely, assume that $x$ is an interior weakly efficient allocation and w.l.o.g. assume that there exists a feasible allocation $y$ such that $y^{1} \succ x^{1}$ and $y^{i} \succeq x^{i}, i \neq 1$. As $x^{1}$ is interior, it is uniformly bounded below. By considering $\frac{\left(y^{1}+x^{1}\right)}{2}$ instead of $y^{1}$, we may also assume that $y^{1}$ is uniformly bounded below.

For each $Q \in \mathscr{P}$, there exists $\varepsilon_{Q}>0$ such that $E^{Q} U^{1}\left(y^{1}-\varepsilon_{Q}\right)>$ $E^{Q} U^{1}\left(x^{1}\right)$ as the map $x \rightarrow E^{Q} U^{1}(x)$ is norm- $L^{\infty}$-continuous. For a given $\varepsilon>0$, let

$$
\mathscr{V}_{\varepsilon}=\left\{Q \in \mathscr{P} \mid E^{Q} U^{1}\left(y^{1}-\varepsilon\right)>E^{Q} U^{1}\left(x^{1}\right)\right\}
$$

As $Q \rightarrow E^{Q} U^{1}\left(y^{1}-\varepsilon\right)-E^{Q} U^{1}\left(x^{1}\right)$ is linear and $L^{1}$-continuous, $\mathscr{V}_{\varepsilon}$ is $\sigma\left(L^{1}, L^{\infty}\right)$ (relatively) open and from the previous argument $\cup_{\varepsilon} \mathscr{V}_{\varepsilon}=\mathscr{P}$. Since $\mathscr{P}$ is compact, there exists a finite subcovering of $\mathscr{P}$ by $\left(\mathscr{V}_{\varepsilon_{i}}\right)$. Let $\varepsilon=\min _{i} \varepsilon_{i}$ and $\varepsilon^{\prime}=\frac{\varepsilon}{I-1}$. We then have

$$
y^{1}-\varepsilon \succ x^{1} \text { and } y^{i}+\varepsilon^{\prime} \succ x^{i}
$$

contradicting the weak efficiency of $x$.

Our main theorem will show that interior allocations are efficient if and only if they are S-efficient in some S-economy. We start with a general 
observation which holds true for all sorts of incomplete preferences that are defined by a family of complete preferences. If an allocation is efficient in some economy with complete preferences in the family, then it is efficient in the economy with incomplete preferences.

Lemma A.3 If there exist priors $Q^{i} \in \mathscr{P}^{i}$ such that $x^{*}$ is efficient in the S-economy with priors $Q=\left(Q^{1}, \ldots, Q^{I}\right)$, then $x^{*}$ is efficient .

Proof: Suppose that $x^{*}$ is efficient in the S-economy with priors $Q=$ $\left(Q^{1}, \ldots, Q^{I}\right)$. From Lemmata A.2 and A.1, $x^{*}$ is efficient if there exists no allocation $y$ such that

$$
E^{P^{i}} U^{i}\left(y^{i}\right)>E^{P^{i}} U^{i}\left(x^{i}\right) \text { for all } i \text { and all } P^{i} \in \mathscr{P}^{i} .
$$

This is obvious since (12) contradicts $x$ being efficient in the S-economy with priors $Q$.

In $\mathrm{S}$-economies, marginal rates of substitution coincide at interior efficient allocations. We recall this fact without proof here.

Lemma A.4 An interior allocation c is efficient in the S-economy with priors $Q=\left(Q^{1}, \ldots, Q^{I}\right)$ if and only if the marginal rates of substitution coincide for all agents,

$$
M R S_{t}^{i}\left(c^{i}, Q^{i}\right)=M R S_{t}^{j}\left(c^{j}, Q^{j}\right), \quad(t=0, \ldots, T, i, j=1, \ldots, I) .
$$

An immediate corollary of the previous two lemmata is the following. When marginal rates coincide for some priors, we have efficiency in the $\mathrm{S}$ economy. But by Lemma A.3, we then also have efficiency in the B-economy.

Corollary A.5 Let c be a feasible, interior allocation. If

$$
\bigcap_{i=1}^{I} \Psi^{i}\left(c^{i}\right) \neq \emptyset,
$$

then $c$ is efficient.

PROOF: If

$$
\bigcap_{i=1}^{I} \Psi^{i}\left(c^{i}\right) \neq \emptyset
$$


then agents' marginal rates of substitution coincide for some priors $Q^{i} \in \mathscr{P}^{i}$. By Lemma A.4, $c$ is efficient in the Savage economy with priors $\left(Q^{1}, \ldots, Q^{I}\right)$. Lemma A.3 yields that $c$ is efficient.

We now move on to the proofs of the lemmata and theorems in the main text.

\section{Proof of Lemma 2.1}

Proof: This lemma is a version of the multiplicative Doob decomposition. Let $\left(c^{i}\right)$ be a feasible, interior allocation, take some $c^{i}$ and write $Z_{t}=M R S_{t}^{i}\left(c^{i}, Q^{i}\right)$. Note that $Z$ is strictly positive, and bounded, because $c^{i}$ is bounded away from zero. Moreover, $Z_{0}=1$.

If we have a decomposition $Z_{t}=M_{t} \exp \left(-\sum_{s=1}^{t} r_{s}\right)$ with a strictly positive martingale $M$ and a predictable process $r$, then we must have for $t=0, \ldots, T-1$

$$
1=E^{P_{0}}\left[\frac{M_{t+1}}{M_{t}} \mid \mathscr{F}_{t}\right]=E^{P_{0}}\left[\frac{Z_{t+1}}{Z_{t}} \mid \mathscr{F}_{t}\right] \exp \left(r_{t+1}\right),
$$

because $r_{t+1}$ is $\mathscr{F}_{t}$-measurable. So the only possible choice is

$$
r_{t+1}=-\log E^{P_{0}}\left[\frac{Z_{t+1}}{Z_{t}} \mid \mathscr{F}_{t}\right]
$$

and

$$
M_{t}=Z_{t} \exp \left(\sum_{s=1}^{t} r_{s}\right)
$$

A straightforward calculation shows that $M$ is a martingale with $M_{0}=Z_{0}=$ 1 .

\section{Proof of Theorem 2.2}

Proof: 3. is equivalent to 2. Let us first show that 3 implies 2. Let $Q \in \bigcap_{i=1}^{I} \Pi^{i}\left(c^{i}\right)$ and denote by $\left(q_{t}\right)$ the corresponding density process with respect to $P_{0}$. Let $r_{t}=r^{i}\left(Q, c^{i}\right)_{t}$ be the common interest rate. Then we have for all $\mathrm{i}$

$$
Q=Q^{i}\left(c^{i}\right)
$$

for some $Q^{i} \in \mathscr{P}^{i}$ and hence

$$
M R S_{t}^{i}\left(c^{i}, Q^{i}\right)=M R S_{t}^{j}\left(c^{j}, Q^{j}\right)
$$


or

$$
\bigcap_{i=1}^{I} \Psi^{i}\left(x^{i}\right) \neq \emptyset
$$

Conversely if $\bigcap_{i=1}^{I} \Psi^{i}\left(x^{i}\right) \neq \emptyset$ or equivalently if $M R S_{t}^{i}\left(c^{i}, Q^{i}\right)=$ $M R S_{t}^{j}\left(c^{j}, Q^{j}\right)$ for all $i, j=1, \ldots, I$ and $t=0, \ldots, T$ from Lemma 2.1, the martingales and subjective interest rates $r^{i}$ coincide. Hence the agents share a risk-adjusted prior $\bigcap_{i=1}^{I} \Pi^{i}\left(x^{i}\right) \neq \emptyset$ and $r^{i}\left(Q, c^{i}\right)_{t}=r^{j}\left(Q, c^{j}\right)_{t}$ for all $i, j=1, \ldots, I$ and $t=0, \ldots, T$

2. implies 4. follows from Lemma A.4.

4. implies 1. follows from Lemma A.3.

Let us now show that 1 . implies 2 .

We will work on the product probability space $(S, \mathscr{S}, \nu)$ given by

$$
S=\Omega \times\{0, \ldots, T\}, \mathscr{S}=\mathscr{A}, \nu=P_{0} \otimes \zeta,
$$

where we recall that $\mathscr{A}$ is the $\sigma$-field generated by all adapted processes and $\zeta$ the uniform probability measure on $\{0, \ldots, T\}$.

Take an interior efficient allocation $\left(c^{i}\right)$ and form the sets

$$
H^{i}:=\Phi^{i}\left(c^{i}\right):=\left\{\left(\frac{M R S_{t}^{i}\left(c^{i}, Q^{i}\right)}{\int_{S} M R S^{i}\left(c^{i}, Q^{i}\right) d \nu}\right)_{t=0, \ldots, T} \mid Q^{i} \in \mathscr{P}^{i}\right\} .
$$

If we treat the product space $S$ as our basic state space, these sets are the risk-adjusted priors as in Rigotti and Shannon (2005). Note that the ratios are well-defined because $\left(c^{i}\right)$ is an interior allocation. The same argument as in Rigotti and Shannon (2005), Lemma 3, Appendix shows that $H^{i}$ is convex.

$H^{i}$ is $\sigma\left(L^{1}(S, \mathscr{S}, \nu), L^{\infty}(S, \mathscr{S}, \nu)\right)$-compact because the marginal utilities are bounded above and below and $\mathscr{P}^{i}$ is $\sigma\left(L^{1}(S, \mathscr{S}, \nu), L^{\infty}(S, \mathscr{S}, \nu)\right)$ compact.

Now suppose $\bigcap_{i} H^{i}=\emptyset$. Samet's Separation Theorem (see Lemma A.6 below for our infinite-dimensional context) then implies that there exist $\left(g^{i}\right)_{i=1}^{n} \in\left(L^{\infty}(S, \mathscr{S}, \nu)\right)^{n}$ with $\sum_{i} g^{i}=0$ such that $\int_{S} h^{i} g^{i} d \nu>0$ for all $h^{i} \in H^{i}$ and all $i$. Let $d^{i}=c^{i}+\lambda g^{i}$ with $\lambda>0$. For $\lambda$ small enough, the allocation $\left(d^{i}\right)$ is feasible and Pareto-dominates $\left(c^{i}\right)$. Indeed, for any $Q^{i} \in \mathscr{P}^{i}$,

$E^{Q^{i}}\left(U_{i}\left(d^{i}\right)-U_{i}\left(c^{i}\right)\right) \geq \lambda \int_{S} M R S_{u}^{i}\left(d^{i}, Q^{i}\right) \nu(d u) \frac{\int_{S} M R S_{u}^{i}\left(d^{i}, Q^{i}\right) g^{i} \nu(d u)}{\int_{S} M R S_{u}^{i}\left(d^{i}, Q^{i}\right) \nu(d u)}>0$ 
for $\lambda$ small enough. This is a contradiction to $c$ being efficient. Therefore, we have $\bigcap_{i} H^{i} \neq \emptyset$, and we can find priors $Q^{i} \in \mathscr{P}^{i}$ such that

$$
\frac{M R S_{t}^{i}\left(c^{i}, Q^{i}\right)}{\int_{S} M R S_{u}^{i}\left(c^{i}, Q^{i}\right) \nu(d u)}=\frac{M R S_{t}^{j}\left(c^{j}, Q^{j}\right)}{\int_{S} M R S_{u}^{j}\left(c^{j}, Q^{j}\right) \nu(d u)}
$$

for all $i, j=1, \ldots, I$ and all $t=0, \ldots, T$ a.s. For $t=0$, the marginal rates of substitution are 1 , so we get

$$
\int_{S} M R S_{u}^{i}\left(c^{i}, Q^{i}\right) \nu(d u)=\int_{S} M R S_{u}^{j}\left(c^{j}, Q^{j}\right) \nu(d u)
$$

for all agents $i, j$. This implies

$$
M R S_{t}^{i}\left(c^{i}, Q^{i}\right)=\operatorname{MR} S_{t}^{j}\left(c^{j}, Q^{j}\right)
$$

and hence

$$
\bigcap_{i=1}^{I} \Psi^{i}\left(x^{i}\right) \neq \emptyset
$$

Let us prove here the version of Samet's Theorem for infinite-dimensional spaces that we used above ${ }^{17}$.

Lemma A.6 (Samet's Separation Theorem for $L^{\infty}$ ) On a probability space $(S, \mathscr{S}, \nu)$, let $\left(H^{i}\right)_{i=1}^{n}$ be nonempty, convex, and $\sigma\left(L^{1}(S, \mathscr{S}, \nu), L^{\infty}(S, \mathscr{S}, \nu)\right)$-compact subsets of densities in $L_{+}^{1}(S, \mathscr{S}, \nu)$. Then $\bigcap_{i=1}^{n} H^{i}=\emptyset$ if and only if there exists $\left(g^{i}\right)_{i=1}^{n} \in\left(L^{\infty}(S, \mathscr{S}, \nu)\right)^{n}$ with $\sum_{i} g^{i}=0$ such that $\int_{S} h^{i} g^{i} d \nu>0$ for all $h^{i} \in H^{i}$ and all $i$.

Proof: We write $L^{\infty}$ for $L^{\infty}(S, \mathscr{S}, \nu)$ and $L^{1}$ for $L^{1}(S, \mathscr{S}, \nu)$ and $E g$ for $\int_{S} g d \nu$. Assume that $\bigcap_{i=1}^{n} H^{i}=\emptyset$ and let $H=H^{1} \times H^{2} \ldots \times H^{n}$ and $L=$ $\left\{(h, h, \ldots, h), h \in L^{1}\right\} . H$ is $\sigma\left(L^{1}\left((S, \mathscr{S}, \nu)^{n}\right), L^{\infty}\left((S, \mathscr{S}, \nu)^{n}\right)\right)$-compact and convex as a product of $\sigma\left(L^{1}, L^{\infty}\right)$-compact and convex sets and $L$ is a norm-closed vector subspace, hence $\sigma\left(L^{1}\left((S, \mathscr{S}, \nu)^{n}\right), L^{\infty}\left((S, \mathscr{S}, \nu)^{n}\right)\right)$ closed. From the separation theorem for convex sets, there exists $c \in$ $\mathbb{R},\left(f^{i}\right)_{i=1}^{n} \in\left(L^{\infty}\right)^{n}$ such that

$$
\sum_{i} E\left(f^{i} h^{i}\right)>c \geq E\left(h \sum_{i} f^{i}\right) \text { for all } h \in L^{1} h^{i} \in H^{i}, i=1, \ldots, n
$$

\footnotetext{
${ }^{17}$ This version of Samet's theorem may also be obtained as a corollary of Theorem 2 in Billot, Chateauneuf, Gilboa, and Tallon (2000), but the proof given here is more direct and much simpler
} 
From the right hand side of the inequality, we obtain since $L$ is a subspace that $c \geq 0=E\left(h \sum_{i} f^{i}\right)$ for all $h \in L^{1}$. Hence $\sum_{i} f^{i}=0$ a.e. From the left hand side, one obtains that $\sum_{i} E\left(f^{i} h^{i}\right)>0$ for all $h^{i} \in H^{i}, i=1, \ldots, n$. Since $h \rightarrow E\left(f^{i} h\right)$ is $\sigma\left(L^{1}, L^{\infty}\right)$-continuous and $H^{i}$ is $\sigma\left(L^{1}, L^{\infty}\right)$-compact, there exists $\bar{h}^{i}$ minimizing $E\left(f^{i} h^{i}\right)$ on $H^{i}$. Since $\sum_{i} E\left(f^{i} \bar{h}^{i}\right)>0$ for all $i$, there exists $\left(m^{i}\right)_{i=1}^{n} \in \mathbb{R}^{n}$ such that $\sum_{i} m^{i}=0$ and $E\left(f^{i} \bar{h}^{i}\right)+m^{i}>0$ for all $i$. Let $g^{i}=f^{i}+m^{i}$. We then have $E\left(g^{i} \bar{h}^{i}\right)=E\left(f^{i} \bar{h}^{i}\right)+m^{i}>0$ and therefore for all $h^{i} \in H^{i}$

$$
E\left(g^{i} h^{i}\right)=E\left(f^{i} h^{i}\right)+m^{i} \geq E\left(f^{i} \bar{h}^{i}\right)+m^{i}>0
$$

and $\sum_{i} g^{i}=\sum_{i} g^{i}+\sum_{i} m^{i}=0$ proving one direction of the lemma. The reverse direction is trivially true.

\section{Proof of Proposition 2.3}

Proof: Let $\left(x^{*}, p^{*}\right)$ be an equilibrium for a S-economy with priors $Q^{i} \in \mathscr{P}^{i}, i=1, \ldots, I$. Suppose that $y^{i} \succ^{i} x^{* i}$ and $p . y^{i} \leq p . \omega^{i}$. As already proven, w.l.o.g. we may assume that $E^{Q^{i}} U^{i}\left(y^{i}\right)>E^{Q^{i}} U^{i}\left(x^{* i}\right)$ for any $Q^{i} \in \mathscr{P}^{i}$. Hence $p . y^{i}>p \cdot \omega^{i}$ contradicting the hypothesis that $\left(x^{*}, p^{*}\right)$ is an equilibrium for the S-economy with priors $\left(Q^{i}\right)$. The proof for equilibria with transfer payments is similar. To prove the second claim, from Theorem 2.2, any interior efficient B-allocation $\left(x^{*}\right)$ is an efficient allocation, hence an equilibrium with transfer payments for some S-economy with priors $Q^{i} \in \mathscr{P}^{i}, i=1, \ldots, I$. From assertion one, $\left(x^{*}\right)$ is a B-equilibrium with transfer payments. To prove the third claim, let $\left(x^{*}, p^{*}\right)$ be an interior B-equilibrium. By the first welfare theorem, $x^{*}$ is efficient. Assume that $\lambda p^{*} \notin H^{i}$ for any $\lambda \geq 0$ where $H^{i}$ is defined in the proof of 4 implies 1 of Theorem 2.2. Since $H^{i}$ is $\sigma\left(L^{1}((S, \mathscr{S}, \nu)), L^{\infty}((S, \mathscr{S}, \nu))\right.$-compact and convex and $p^{*} \lambda$ for $\lambda \geq 0$ is a $\sigma\left(L^{1}\left((S, \mathscr{S}, \nu), L^{\infty}(S, \mathscr{S}, \nu)\right)\right.$-closed convex cone, from the separation theorem for a convex closed cone and a convex compact set, there exists $f^{i} \in L^{\infty}$ such that

$$
p^{*} \dot{f}^{i} \leq 0<\min _{H^{i}} f^{i} \dot{h}^{i}
$$

Using again the proof of Theorem 2.2, $d^{i}=x^{* i}+\mu f^{i}$ with $\mu>0$ small enough is such that $d^{i} \succ x^{* i}$ while $p^{*} \dot{d}^{i} \leq p^{*} \dot{x}^{* i}$ contradicting the hypothesis that $p^{*}$ is a B-equilibrium price. Hence $\frac{p^{*}}{\int_{S} p^{*} d \nu} \in H^{i}$. Since this is true for each agent, $\cap H^{i} \neq \emptyset$ and $\frac{p^{*}}{\int_{S} p^{*} d \nu} \in \cap H^{i}$. From the proof of Theorem 2.2, we can find 
priors $Q^{i} \in \mathscr{P}^{i}$ such that

$$
p_{t}^{*}=a M R S_{t}^{i}\left(c^{i}, Q^{i}\right), a>0
$$

for all $i=1, \ldots, I$ and all $t=0, \ldots, T$ a.s. which implies that $\left(x^{*}, p^{*}\right)$ is an equilibrium for the S-economy with priors $\left(Q^{i}\right)$.

\section{Proof of Corollary 2.4}

Proof: Let $Q \in \bigcap_{i=1}^{I} \mathscr{P}^{i}$. Then the S-economy with common prior $Q$ and no aggregate risk has a full insurance equilibrium. Corollary 2.4 then follows from assertion 3 of Proposition 2.3.

\section{Proof of Theorem 2.6}

PROOF: In order to prove existence of an equilibrium with inertia, let

$$
V^{i}(x)=\min _{P \in \mathscr{P}^{i}} E^{P}\left(U^{i}(x)-U^{i}\left(\omega^{i}\right)\right) .
$$

By Lemma A.8 in the next section, these preferences are Mackey-continuous. Hence, the existence theorem of Bewley (1972) may be applied to get an equilibrium $(\bar{p}, \bar{x})$ with strictly positive price for the economy with complete preferences $\left(V^{i}\right)$ which will be referred to as the $\mathrm{V}$-economy. Let us show that $(\bar{p}, \bar{x})$ is an equilibrium in the $\mathrm{B}$-economy. Budget constraints are trivially fulfilled. Let $x^{i} \succ \bar{x}^{i}$. W.l.o.g, as we already argued, we may assume that $E^{Q} U^{i}\left(x^{i}\right)>E^{Q} U^{i}\left(\bar{x}^{i}\right)$ for any $Q \in \mathscr{P}^{i}$. Hence $V^{i}\left(x^{i}\right)>V^{i}\left(\bar{x}^{i}\right)$ and therefore $\bar{p} . x^{i}>\bar{p} . \bar{x}^{i}$ proving that $(\bar{p}, \bar{x})$ is an equilibrium in the $\mathrm{B}$-economy. Let us now show that any equilibrium in the $\mathrm{V}$-economy is an equilibrium in the B-economy with inertia. We claim that any equilibrium allocation in the $\mathrm{V}-$ economy $\bar{x}$ verifies $V^{i}\left(\bar{x}^{i}\right)>V\left(\omega^{i}\right)=0$ for any $i$ such that $\bar{x}^{i} \neq \omega^{i}$. If not, $V^{i}$ being strictly concave $V^{i}\left(\left(\bar{x}^{i}+\omega^{i}\right) / 2\right)>V\left(\omega^{i}\right)=0$ while $\bar{p} \cdot\left(\bar{x}^{i}+\omega^{i}\right) / 2=\bar{p} \cdot \bar{x}^{i}$ a contradiction to $(\bar{p}, \bar{x})$ being an equilibrium of the $\mathrm{V}$-economy. Therefore $E^{Q} U^{i}\left(\bar{x}^{i}\right)>E^{Q} U^{i}\left(\omega^{i}\right)$ for any $i$ such that $\bar{x}^{i} \neq \omega^{i}$ and any $Q \in \mathscr{P}^{i}$. Thus $\bar{x}^{i} \succ^{i} \omega^{i}$. Hence any equilibrium in the economy with preferences $\left(V^{i}\right)$ is an equilibrium with inertia.

\section{Proof of Corollary 2.8}

Proof: If $\left(\left(\omega^{i}\right), p\right)$ is an equilibrium of the $\mathrm{V}$-economy, then from Theorem 2.6 , it is an equilibrium of the B-economy. We claim that $\left(\omega^{i}\right)$ is the unique Bequilibrium allocation with inertia. Let $\left(x^{i}\right)$ be another equilibrium allocation with inertia. Then we either have $x^{i}=\omega^{i}$ or $x^{i} \neq \omega^{i}$ and in that case additionally $x^{i} \succ \omega^{i}$. For these agents $i$, we have $p . x^{i}>p . \omega^{i}$ because $p$ is an equilibrium price. Summing up over agents, we obtain a contradiction. 
Conversely, if $\left(\omega^{i}\right)$ is the unique equilibrium allocation in the B-economy, then it is efficient and since $\left(\omega^{i}\right)$ is an interior allocation, $\bigcap_{i} \Phi^{i}\left(\omega^{i}\right) \neq \emptyset$ (where $\Phi^{i}$ is defined in the proof of Theorem 2.2). Hence, $\left(\omega^{i}\right)$ is efficient in the $\mathrm{V}$-economy since any $Q \in \mathscr{P}^{i}$ is a minimizing probability for $V^{i}$ at $\omega^{i}$ (by definition of $\left.V^{i}\right)$. Since $\left(\omega^{i}\right)$ is an equilibrium allocation in the B-economy, there exists a price process $p \in \mathbb{P}$ such that $E^{Q}\left(U^{i}(x)\right)>E^{Q}\left(U^{i}\left(\omega^{i}\right)\right)$ for all $Q \in P^{i}$ implies $p . x^{i}>p . \omega^{i}$. Hence $V^{i}(x)>V^{i}\left(\omega^{i}\right)=0$ implies $p . x>p . \omega^{i}$ and therefore $\left(\omega^{i}\right)$ is an equilibrium allocation in the $\mathrm{V}$-economy.

\section{A.2 Mackey-Continuity of Variational Preferences Anchored at Endowments}

Lemma A.8 which shows Mackey-continuity of a special variational preference introduced in the paper, extends a proof in Dana (2002). The reader may verify that this proof does not extend to general variational preferences with a lower semi-continuous penalty function. The following estimates for utility increments will be useful for Lemma A.8.

Lemma A.7 Let $u: \mathbb{R}_{+} \rightarrow \mathbb{R}$ be strictly concave and strictly increasing, $C^{1}$, and verify $u(0)=0$. Then for any $\eta>0, x, y \in \mathbb{R}_{+}$, we have

$$
|u(x)-u(y)| \leq \frac{u(\eta)}{\eta}|x-y|+2 u(\eta)
$$

Proof: Let $\eta>0$ be given. If $x<\eta$ and $y>\eta$,

$$
\frac{|u(y)-u(x)|}{|y-x|}=\frac{u(y)-u(x)}{y-x} \leq \frac{u(y)}{y} \leq \frac{u(\eta)}{\eta}
$$

by concavity and monotonicity. If $x<\eta$ and $y<\eta,|u(x)-u(y)| \leq 2 u(\eta)$. Hence if $x<\eta$, we have

$$
|u(x)-u(y)| \leq \frac{u(\eta)}{\eta}|x-y|+2 u(\eta) .
$$

If $x>\eta$ and $y<\eta$, we similarly have $|u(x)-u(y)| \leq \frac{u(\eta)}{\eta}|x-y|$ while if $x>\eta$ and $y>\eta,|u(x)-u(y)| \leq u^{\prime}(\theta)|x-y|$ for some $\left.\theta \in\right] x, y[$. Hence

$$
|u(x)-u(y)| \leq \frac{u(\eta)}{\eta}|x-y|
$$


proving (13) .

Let $\tau\left(L^{\infty}, L^{1}\right)$ denote the Mackey topology on $L^{\infty}\left(\Omega \times\{0, \ldots, T\}, \mathscr{A}, P_{0} \otimes \zeta\right)$.

Lemma A.8 Let $U: \mathbb{R}_{+}{ }^{\{0, \ldots, T\}} \rightarrow \mathbb{R}$ be defined by $U(c)=\sum_{t=0}^{T} \rho^{t} u\left(c_{t}\right)$ with $u$ strictly increasing, strictly concave and $C^{1}$ and verify $u(0)=0$. Let $\mathscr{P}$ fulfill assumption 2.1. Let $y \in L^{\infty}\left(\Omega \times\{0, \ldots, T\}, \mathscr{A}, P_{0} \otimes \zeta\right)$ be fixed. Then the utility function $V: \mathscr{X}_{+} \rightarrow \mathbb{R}$ defined by

$$
V(x)=\min _{Q \in \mathscr{P}} E^{Q}(U(x)-U(y))
$$

is $\tau\left(L^{\infty}, L^{1}\right)$-continuous, strictly concave and monotone.

Proof: For $Q \in \mathscr{P}$, let $q=\left.\frac{d Q}{d P_{0}}\right|_{\mathscr{F}_{T}}$ denote the time- $T$ density with respect to $P_{0}$ and let $\mathscr{D}$ be the set of densities. Let $x_{\alpha} \stackrel{\tau}{\rightarrow} x$ (or equivalently, let $x_{t_{\alpha}} \stackrel{\tau}{\rightarrow} x_{t}$ for all $t$ ) and $q_{\alpha}$ be such that

$$
V\left(x_{\alpha}\right)=E^{P_{0}}\left[q_{\alpha}\left(U\left(x_{\alpha}\right)-U(y)\right)\right] .
$$

Such an $q_{\alpha}$ exists since $\mathscr{D}$ is $\sigma\left(L^{1}, L^{\infty}\right)$ compact and $\left(U\left(x_{\alpha}\right)-U(y)\right) \in L^{\infty}$. Since $\mathscr{D}$ is $\sigma\left(L^{1}, L^{\infty}\right)$ compact, we may assume w.l.o.g. that $q_{\alpha} \stackrel{\sigma}{\rightarrow} q$. Let us show that the restriction to $L_{+}^{\infty}\left(\Omega \times\{0, \ldots, T\}, \mathscr{A}, P_{0} \otimes \zeta\right) \times \mathscr{D}$ endowed with $\tau\left(L^{\infty}, L^{1}\right) \times \sigma\left(L^{1}, L^{\infty}\right)$ of the map $(x, q) \rightarrow E(q(U(x)-U(y)))$ is jointly continuous. Let $x_{\alpha} \stackrel{\tau}{\rightarrow} x$ and $q_{\alpha} \stackrel{\sigma}{\rightarrow} q$. Let us prove that $E^{P_{0}}\left(q_{\alpha}\left(U\left(x_{t_{\alpha}}\right)-\right.\right.$ $U(x)+\left(q_{\alpha}-q\right)\left(U\left(x_{t}\right)-U(y)\right) \rightarrow 0$. Since $q_{\alpha} \stackrel{\sigma}{\rightarrow} q$, the second term goes to zero. To study the first term, since $x_{t_{\alpha}} \stackrel{\tau}{\rightarrow} x_{t}$ and the Mackey topology is locally solid, $\left|x_{t_{\alpha}}-x_{t}\right| \stackrel{\tau}{\rightarrow} 0$. From Lemma A.7, we have for any $\eta>0$

$$
E^{P_{0}} \mid U\left(x_{t_{\alpha}}-U\left(x_{t}\right) \mid q_{\alpha} \leq U(\eta)\left[2+\frac{1}{\eta} E^{P_{0}}\left(\left|x_{t_{\alpha}}-x_{t}\right| q_{\alpha}\right)\right]\right.
$$

For any $\varepsilon>0$, choose $\eta>0$ such that $U(\eta) \leq \varepsilon$ and $\alpha$ such that $\sup _{q \in \mathscr{D}} E^{P_{0}}\left[\left|x_{t_{\alpha}}-x_{t}\right| q\right]<\eta$, then the above integral is smaller then $3 \varepsilon$, which proves the claimed joint continuity.

Hence

$$
V\left(x_{\alpha}\right)=E^{P_{0}}\left[q_{\alpha}\left(U\left(x_{\alpha}\right)-U(y)\right)\right] \rightarrow E^{P_{0}}[q(U(x)-U(y))] .
$$

By definition of $q_{\alpha}$,

$$
E^{P_{0}}\left[q_{\alpha}\left(U\left(x_{\alpha}\right)-U(y)\right)\right] \leq E^{P_{0}}\left[q\left(U\left(x_{\alpha}\right)-U(y)\right)\right] \text { for all } q \in \mathscr{D} .
$$


In the limit, we obtain

$$
E^{P_{0}}[q(U(x)-U(y))] \leq \min _{q \in \mathscr{D}} E^{P_{0}}[q(U(x)-U(y))]=V(x),
$$

hence

$$
E^{P_{0}}[q(U(x)-U(y))]=\min _{q \in \mathscr{D}} E^{P_{0}}[q(U(x)-U(y))]=V(x),
$$

proving the continuity of $V$ with respect to the Mackey topology.

Let us prove that $V$ is strictly monotone. As the priors are equivalent to the reference probability $P_{0}$, any $q \in \mathscr{D}$ is strictly positive. As $U$ is strictly concave, for any $z \in L_{+}^{\infty}$, we have

$$
V(x+z)-V(x) \geq E^{P_{0}}\left[q_{x+z}(U(x+z)-U(x))\right]>E^{P_{0}}\left[q_{x+z} U^{\prime}(x+z) z\right]>0
$$

where $q_{x+z}$ is a minimizing density for $V(x+z)$. Therefore $V$ is strictly monotone.

Finally, let us show the strict concavity of $V$. Let $\lambda \in] 0,1[$ be given. Then we have

$$
\begin{aligned}
V(\lambda x+(1-\lambda) z) \geq E^{P_{0}}\left[q_{\lambda x+(1-\lambda) z} U(\lambda x+(1-\lambda) z)\right. & \\
& >\lambda E^{P_{0}}\left(q_{x} U(x)+(1-\lambda) q_{z} U(z)\right),
\end{aligned}
$$

since $U$ is strictly concave and $q_{x}$ is a minimizing probability for $V$ at $x$.

\section{A.3 Auxiliary Proofs for Section 3}

\section{Proof of Lemma 3.5}

Proof: Let $Q$ be an agent's prior associated to the process $\left(\alpha_{s}\right)$. Then

$$
\begin{aligned}
& E^{Q}\left(\exp \left(-\gamma U_{t}\right)=E^{P_{0}}\left(\exp \left(\sum_{s=1}^{t}\left(\alpha_{s}-\gamma\right) \nu_{s}-\frac{\alpha_{s}^{2}}{2}\right)\right)\right. \\
& \quad=E\left[E\left[\exp \left(\sum_{s=1}^{t}\left(\alpha_{s}-\gamma\right) \nu_{s}-\frac{\alpha_{s}^{2}}{2}\right) \mid \mathscr{F}_{t-1}\right]\right]
\end{aligned}
$$


As $\left(\alpha_{t}\right)$ is a $\mathscr{F}^{U}$-predictable process, we have

$$
\begin{gathered}
E^{Q}\left(\exp \left(-\gamma U_{t}\right)\right)=E\left[\exp \left(\sum_{s=1}^{t-1}\left(\alpha_{s}-\gamma\right) \nu_{s}-\frac{\alpha_{s}^{2}}{2}\right) \exp \left(\frac{\left(\gamma+\alpha_{t}\right)^{2}}{2}-\frac{\alpha_{t}^{2}}{2}\right)\right] \\
=E\left[\exp \left(\sum_{s=1}^{t-1}\left(\alpha_{s}-\gamma\right) \nu_{s}-\frac{\alpha_{s}^{2}}{2}\right) \exp \left(\frac{\gamma}{2}+\alpha_{t} \gamma\right)\right] \\
\geq E\left[\exp \left(\sum_{s=1}^{t-1}\left(\alpha_{s}-\gamma\right) \nu_{s}-\frac{\alpha_{s}^{2}}{2}\right) \exp \left(\frac{\gamma}{2}-\kappa|\gamma|\right)\right]
\end{gathered}
$$

By induction, we thus obtain that

$$
E^{Q}\left(\exp \left(-\gamma U_{t}\right)\right) \geq \exp \left(\frac{\gamma^{2}}{2}-\kappa|\gamma|\right) t
$$

The upper bound follows from a similar argument.

Lemma A.9 Let $\left(q_{t}\right)$ be the density process of some $Q \in \mathscr{P}$. Then we have

$$
E q_{t}^{2} \leq \exp \left(\kappa^{2} t\right)
$$

Proof: Remember that $q$ can be written as

$$
q_{t}=\exp \left(\sum_{s=1}^{t}\left(\alpha_{s} \nu_{s}-\frac{1}{2} \alpha_{s}^{2}\right)\right)
$$

for some $\mathscr{F}^{U}$-predictable process $\left(\alpha_{t}\right)$ with values in the interval $[-\kappa, \kappa]$. Note that the process

$$
\exp \left(\sum_{s=1}^{t}\left(2 \alpha_{s} \nu_{s}-\frac{1}{2} 4 \alpha_{s}^{2}\right)\right)=\exp \left(\sum_{s=1}^{t}\left(2 \alpha_{s} \nu_{s}-2 \alpha_{s}^{2}\right)\right)
$$

is a martingale with expectation 1 . We can thus estimate

$$
\begin{aligned}
E q_{t}^{2} & =E \exp \left(\sum_{s=1}^{t}\left(2 \alpha_{s} \nu_{s}-\frac{1}{2} 4 \alpha_{s}^{2}+\alpha_{s}^{2}\right)\right) \\
& \leq E \exp \left(\sum_{s=1}^{t}\left(2 \alpha_{s} \nu_{s}-\frac{1}{2} 4 \alpha_{s}^{2}+\kappa^{2}\right)\right) \\
& =\exp \left(\kappa^{2} t\right) .
\end{aligned}
$$




\section{References}

Bewley, T. (1972): "Existence of Equilibria in Economies with Infinitely Many Commodities," Journal of Economic Theory, 4, 514-540.

- (2002): "Knightian Decision Theory: Part I," Decisions in Economics and Finance, 25, 79-110.

Billot, A., A. Chateauneuf, I. Gilboa, and J. Tallon (2000): "Sharing Beliefs: Between Agreeing and Disagreeing," Econometrica, 68, 685694 .

Bryan, G. (2010): "Ambiguity and Insurance," Working Paper, Dept. of Economics, Yale University.

Chen, Z., And L. Epstein (2002): "Ambiguity, Risk and Asset Returns in Continuous Time," Econometrica, 70, 1403-1443.

DanA, R. (2002): "On Equilibria when Agents Have Multiple Priors," Annals of Operations Research, 114, 105-112.

Easley, D., And M. O'Hara (2010): "Liquidity and Valuation in an Uncertain World," Journal of Financial Economics, 97, 1-11.

Epstein, L., And M. MarinaCCI (2007): "Mutual Absolute Continuity of Multiple Priors," Journal of Economic Theory, 137, 716-720.

Epstein, L., And M. Schneider (2003): "Recursive Multiple Priors," Journal of Economic Theory, 113, 1-31.

FAro, J. (2010): "Variational Bewley Preferences," Working Paper.

Gale, D., And A. Mas-Colell (1975): "An Equilibrium Existence Theorem for a General Model without Ordered Preferences," Journal of Mathematical Economics, 2, 9-15.

Gilboa, I., F. Maccheroni, M. Marinacci, and D. Schmeidler (2010): "Objective and Subjective Rationality in a Multiple Prior Model," Econometrica, 78, 755-770.

Maccheroni, F., M. Marinacci, And A. Rustichini (2006): "Ambiguity Aversion, Robustness, and the Variational Representation of Preferences," Econometrica, 74, 1447-1498. 
Minm, M. (2010): "Reference-Dependent Ambiguity Aversion," Working Paper, NYU.

Nascimento, L., And G. Riella (2011): "A Class of Incomplete and Ambiguity Averse Preferences," Journal of Economic Theory, 146, 728750 .

Nehring, K. (2009): "Imprecise Probabilistic Beliefs as a Context for Decision-Making under Ambiguity," Journal of Economic Theory, 144, 1054-1091.

Ok, E., P. Ortoleva, and G. Riella (2012): "Incomplete Preferences under Uncertainty: Indecisiveness in Beliefs versus Tastes," Econometrica, 80, 1791-1808.

RIEDEL, F. (2009): "Optimal Stopping with Multiple Priors," Econometrica, $77,857-908$.

Rigotti, L., and C. Shannon (2005): "Uncertainty and Risk in Financial Markets," Econometrica, 73(1), 203-243.

Rigotti, L., C. Shannon, and T. Strzalecki (2008): "Subjective Beliefs and ex-ante Trade," Econometrica, 76, 1167-1190. 